\title{
Fatores biomecânicos: uma análise do trabalho dos enfermeiros
}

\author{
Marcos Lucas de Oliveira \\ Cristiane Jordão \\ Lizandra Garcia Lupe Vergara
}

\begin{abstract}
Resumo: Os fatores humanos e a ergonomia têm como cerne o domínio dos cuidados de saúde com foco na maximização do desempenho global dos sistemas. As atuais pesquisas sobre saúde e segurança do paciente têm centrado esforços na amplitude do termo resiliência do sistema. Assim, dado o aumento de pesquisas de intervenção ergonômica na área da saúde e da importância dos fatores humanos e da ergonomia no âmbito hospitalar, este estudo tem como objetivo apresentar os principais problemas biomecânicos relacionados à atividade dos enfermeiros, por meio de uma revisão básica da literatura. Como resultado, o estudo apresenta um dossiê dos estudos mais relevantes sobre riscos biomecânicos na atividade laboral dos enfermeiros. Conclui-se que no setor da saúde no Brasil, não há ainda uma dispersão em grande escala dos equipamentos que visam prevenir os riscos biomecânicos relacionados à atividade laboral dos profissionais da saúde.
\end{abstract}

Palavras-chave: Ergonomia; Biomecânica; DORT.

\section{Biomechanical factors: an analysis of the work of nurses}

Abstract: Human factors and ergonomics have as their core the domain of health care with a focus on maximizing the overall performance of systems. Current research on patient health and safety has focused efforts on the breadth of the term system resilience. Thus, given the increase of research on ergonomic intervention in the area of health and the importance of human factors and ergonomics in the hospital environment, this study aims to present the main biomechanical problems related to nurses' activity, through a basic review of the literature. As a result, the study presents a dossier of the most relevant studies on biomechanical risks in nurses' work activity. It is concluded that to the health sector in Brazil, there is not yet a largescale dispersion of equipment aimed at preventing the biomechanical risks related to the work activity of health professionals.

Keywords: Ergonomics; Biomechanics; Spinal card injury.

Factores biomecánicos: análisis del trabajo de los enfermeros

Resumes: Los factores humanos y la ergonomía tienen como núcleo el dominio de los cuidados de la salud con un enfoque en maximizar el rendimiento general de los sistemas. Las actuales investigaciones sobre salud y seguridad del paciente han centrado los esfuerzos en la amplitud del término resiliencia del sistema. De este modo, dado el aumento de las investigaciones sobre intervención ergonómica en el área de la salud y de la importancia de los factores humanos y de la ergonomía en el entorno hospitalario, este estudio pretende presentar los principales problemas biomecánicos relacionados con la actividad de los enfermeros, a través de una revisión básica de la literatura. Como resultado, el estudio presenta un dossier de los estudios más relevantes sobre riesgos biomecánicos en la actividad laboral de los enfermeros. Se concluye que en el sector salud en Brasil, aún no hay una dispersión en gran nivel de equipos para prevenir riesgos biomecánicos relacionados con la actividad laboral de los profesionales de la salud.

Palabras clave: Ergonomía; Biomecánica; DORT.

\section{Introdução}


Distúrbios osteomusculares relacionados ao trabalho (DORT), como dores nas costas e em membros superiores, são as principais causas de distúrbios relacionados ao trabalho e doenças ocupacionais na maioria dos países (PARENT-THIRION et al., 2012; ROQUELAURE, 2016). Nesse contexto, os traumas musculares são citados na legislação brasileira por meio da norma regulamentadora (NR17), que referencia a ergonomia (ATLAS, 2016), passando a ser enquadrado nessa norma como DORT.

Segundo Iida e Buarque (2017), os traumas musculares são provocados pela incompatibilidade entre as exigências do trabalho e as capacidades físicas do trabalhador. Neste aspecto, revendo o desenvolvimento do tema, mais especificamente, para a área da saúde, notase que a inserção de pesquisas sobre DORT é relativamente menor do que a maioria do setor de prestação de serviços (RATHORE; ATTIQUE e ASMAA, 2016).

Moazzami et al. (2015) argumentam que os afastamentos de trabalho por DORT são comuns aos profissionais da área saúde, como médicos, enfermeiros, fisioterapeutas e técnicos. $\mathrm{O}$ autor declara, ainda, que são poucos os estudos na área da saúde que apresentam as causas desses afastamentos. Os principais apontam que a DORT, nesses profissionais, está ligada aos seguintes fatores: (i) postura inadequada de trabalho; (ii) postura em pé por longo período e (iii) postura fixa, segurando e manuseando equipamentos (SHEIKHZADEH et al., 2009; MOAZZAMI et al., 2015).

Para a Organização Mundial de Saúde (OMS), a DORT está relacionada com os problemas de saúde do aparelho locomotor, podendo serem músculos, tendões, esqueleto, cartilagem, ligamentos e nervos (RATHORE, ATTIQUE e ASMAA, 2016). Os fatores de risco da DORT incluem atividades no local de trabalho, como elevação de carga pesada, tarefas repetitivas e posturas de trabalho inadequadas (VANDERGRIFT et al., 2012). Além disso, levam em consideração as variáveis preditivas que são as características demográficas e os fatores psicossociais (D’ERRICO et al., 2010; CHANCHAI et al., 2016).

Nesse contexto, os profissionais da saúde, que preenchem a lacuna entre médicos e pacientes, devido à natureza de seu trabalho, estão propensos a DORT (RATHORE; ATTIQUE e ASMAA, 2016). Estudos em países da Oceania mostraram que a prevalência média de DORT entre enfermeiros é de cerca de 84\%, com a região lombar (RATHORE; ATTIQUE e ASMAA, 2016). Conforme Chanchai et al. (2016), as atividades de manuseio do paciente, por exemplo, submetem os enfermeiros e os técnicos de enfermagem a altas cargas biomecânicas. Dessa forma, o presente estudo tem como objetivo verificar quais são os principais problemas biomecânicos relacionados à atividade laboral dos enfermeiros, por meio de uma revisão básica da literatura. 


\section{Referencial Teórico}

Esta etapa do artigo visa apresentar os conceitos que se relacionam ao objetivo do trabalho. Primeiramente, trata-se de uma breve contextualização referente à ergonomia, seguida das óticas de diferentes autores quanto aos fatores biomecânicos na organização do trabalho e as suas principais particularidades e a evolução dos seus conceitos. Por fim, aborda-se os principais impactos da carga postural e os distúrbios osteomusculares relacionados ao trabalho.

\subsection{Ergonomia}

Desde a sua origem, na década de 1950, a ergonomia passou a realizar estudos cada vez mais abrangentes sobre o trabalho humano (HENDRICK, 1991; IIDA e BUARQUE, 2016). Em sua evolução histórica, a ergonomia pode ser classificada em quatro fases: (i) entre 1950-1960, refere-se à fundação da ergonomia e está voltada a ergonomia física, os seus estudos restringemse ao binômio humano-máquina; (ii) em meados de 1970, destina-se à incorporação das variáveis do meio ambiente (iluminação, temperatura, ruído); (iii) em 1980, refere-se aos estudos cognitivos (percepção, procedimentos de informações, tomada de decisões) do trabalho; e (iv) em 1990, trata da ergonomia organizacional ou macroergonomia, nessa fase, inseriram-se os aspectos organizacionais e gerenciais do trabalho (IIDA e BUARQUE, 2016).

A ergonomia está preocupada com a compreensão das interações entre humanos e outros elementos de um sistema, a fim de otimizar o bem-estar humano e o desempenho geral de um sistema (IEA, 2017). Dul e Neumann (2009) e Tortorella, Vergara e Ferreira (2016) descrevem em seus estudos que a definição de ergonomia transcende a três implicações principais: (i) objetivos sociais e econômicos, (ii) aspectos humanos físicos e psicológicos, e (iii) melhorias nos domínios técnico e organizacionais.

Nessa linha de argumentação, o conceito proeminente que acentua a influência crucial dos fatores sociais e organizacionais em projetos de sistemas de trabalho e processos seguros e operacionais é a macroergonomia. Hendrick (2008) define a macroergonomia como uma abordagem de um sistema sócio-técnico para o projeto de estruturas organizacionais e de sistemas de trabalho. Esses autores também definem-na como sendo a aplicação de um projeto geral de trabalho ao sistema de empregos individuais e interfaces homem-máquina e humanosoftware (AZADEH; ROUDI e SALEHI, 2017). 
A macroergonomia destaca a natureza crucial dos fatores organizacionais na concepção de processos criativos e sistemas de trabalho seguros. Assim, exerce um impacto vital sobre fatores humanos tradicionais e ergonômicos (HENDRICK; KLEINER, 2002). Azadeh et al. (2015) apontam que o estudo da macroergonomia aprimora o desempenho dos sistemas sóciotécnicos e auxilia a compreender as influências das estruturas organizacionais sobre a segurança e comportamento humano. Assim sendo, para entender um estudo macroergonômico, é necessário, primeiro, analisar as dimensões-chave da estrutura do sistema de trabalho (AZADEH; ROUDI e SALEHI, 2017).

Conforme os autores Hollnagel (2012) e Karsh, Waterson e Holden (2013), deve-se agir de forma proativa e não apenas com base em lesões musculares decorridas aos profissionais por meio do histórico de afastamentos. É essencial conhecer os riscos ergonômicos para incentivar a tomada de decisões de forma assertiva ao lidar com eles, ou mesmo para antecipar eventos futuros que trazem riscos potenciais (WOODS, 2015).

\subsection{Distúrbios osteomusculares na organização do trabalho}

Os distúrbios osteomusculares são fatores de relevância quanto à investigação nos ambientes de trabalho (SHIGEYUKI et al., 2017). Dentre eles, a dor lombar é uma das doenças mais comuns e afeta em torno de $30 \%$ da população (VANDERGRIFT et al., 2011). No Brasil, a lombalgia é a principal causa de afastamento no trabalho, por mais de quinze dias, dentre as doenças ocupacionais (BRASIL, 2018; PAULA et al., 2016). Na Europa e nos EUA, a lombalgia também é a principal causa dos afastamentos de trabalho (D’ERRICO et al., 2013).

Os fatores que estão ligados à etiologia da lombalgia estão associados principalmente ao local de trabalho (PUNNETT et al., 2005; VANDERGRIFT et al., 2011). Para Okunribido, Magnusson e Pope (2008), as exposições ergonômicas físicas ocupacionais, como: (i) atividade com posturas posteriores incômodas; (ii) esforço manual relacionado ao manuseio de objetos e (iii) exposição à vibração do corpo, estão associadas ao risco de lombalgia. Além disso, por meio da teoria da biomecânica, é possível inferir que há possibilidade de aumento do risco de lombalgias quando o empregado exerce uma atividade que exija esforço físico combinado a uma postura inadequada (IIDA e BUARQUE, 2017).

Essa teoria, ainda, adverte que a fadiga muscular local, resultante do esforço físico relacionado à tarefa, pode aumentar o risco de lombalgia (IIDA e BUARQUE, 2017). Neste sentido, os fatores ergonômicos de risco físico e psicossociais são frequentemente correlacionados entre si no ambiente de trabalho (HARCOMBE et al., 2010; VANDERGRIFT et 
al., 2011). A exposição ao estresse psicossocial é descrito como um dos fatores de influência à etimologia da lombalgia. De acordo com Waters et al. (2007), estudos experimentais e epidemiológicos sugerem que as exposições a trabalhos que exijam esforços físicos e psicossociais podem interferir sinergicamente em traumas musculares.

Nesse âmbito, os profissionais da área da saúde são a classe de trabalhadores que apresentam uma das maiores taxas de incidência de distúrbios osteomusculares relacionados ao trabalho (DANIELL; MERRETT e PAUL, 2014). A lombalgia entre os profissionais de saúde foi identificada como propulsora do aumento de lesão ocupacionais em comparação com outras profissões (WATERS; NELSON e PROCTOR, 2007; SZETO et a1., 2012). No entender de Daniell, Merrett e Paul (2014), a movimentação de macas e o transbordo de pacientes pelos profissionais da saúde são identificados como as causas mais comuns aos afastamentos por dores musculoesqueléticas. Choi e Brings (2016) complementam afirmando que essas duas atividades constituam uma pequena parte de todas as tarefas, desenvolvidas por esses profissionais, que possuem potencial para causar distúrbios osteomusculares.

Para Weiner (2017), a principal tarefa causadora das lesões ocupacionais aos enfermeiros e aos técnicos de enfermagem refere-se ao reposicionamento do corpo do paciente ao leito. Essa atividade inclui as tarefas de manuseio, transporte ou suporte manual de uma carga. Na ótica da biomecânica, essa atividade infere ao profissional na execução de uma força corpórea para levantar, abaixar, empurrar, puxar, transportar ou movimentar, usando as mãos, o paciente (DOUGHERTY e LISTER, 2011).

\subsection{Fatores musculoesqueléticos relacionados ao trabalho dos enfermeiros}

A Instrução Normativa $\mathrm{n}^{\mathrm{o}} 98$ do Ministério da Saúde define transtornos musculoesqueléticos como um conjunto de sinais e sintomas que resultam em dor, limitação do movimento, parestesia, sensação de peso, fadiga e incapacidade ao trabalho (BRASIL, 2003), sendo esses fatores passíveis de surgirem isoladamente ou simultaneamente. Weiner et al. (2017) ressalvam que as lesões musculoesqueléticas relacionadas ao trabalho são associados à nomenclatura de riscos ergonômicos e estão propensas às atividades laborais que decorrem com: (i) posturas inadequadas, estáticas ou repetidas; (ii) flexão interativa ou sustentada do tronco; (iii) levantamento de cargas e/ou materiais, acima do nível do ombro ou abaixo da altura do joelho; e (iv) manuseio de atividades que exijam rotação ou movimentos combinados.

Para Lee, Dziadkowiec e Meek (2014), os profissionais da saúde, como enfermeiros, estão expostos a riscos musculoesqueléticos por atuarem em um ambiente de trabalho de 
natureza complexa e de atividades multifacetadas. Conforme Santos et al. (2017), as principais regiões anatômicas afetadas ao enfermeiros, em vista do desequilíbrio entre as repetidas demandas mecânicas do trabalho e da capacidade insuficiente de adaptação da zona corporal atingida, são: (i) lombar; (ii) joelhos; (iii) ombros e (iv) cervical. No exercício de suas funções, os enfermeiros são suscetíveis à fadiga ocupacional e à DORT por envolverem tarefas que exijam posturas inadequadas e pela contínua necessidade de movimento dos membros superiores (LIMA et al., 2014).

Nesse contexto, a fadiga ocupacional pode ser definida como um estado multidimensional que surge em trabalhadores expostos a exigências excessivas, por meio de tarefas, ambientes e horários de trabalho, que podem interferir nas habilidades físicas e cognitivas dos trabalhadores e em sua capacidade normal de funcionar (STEEGE et al., 2015; STEEGE e DYKSTRA, 2015). Lee, Dziadkowiec e Meek (2014) descrevem que, em um estudo de fadiga ocupacional, deve-se considerar: (i) os fatores individuais, como idade, ritmo cardíaco e experiência de trabalho anterior; e (ii) o sistema de trabalho, isto é, se envolve tarefas repetitivas e trabalhos por turnos.

No ambiente hospitalar, a fadiga prevalente em enfermeiros é substancial e inclui: (i) o aumento dos eventos adversos do paciente, como quedas e erros de medicação; e (ii) causas individuais, como diminuição de reação a respostas rápidas para tomada de decisões, diminuição da produtividade, problemas de saúde, riscos de acidentes do trabalho e possível aumento da rotatividade de função proveniente da insatisfação com o trabalho (WILLIAMSON e FRISWELL, 2013; BAE e FABRY, 2014). Essas situações podem refletir em graus variados de incapacidade funcional, que refletem, consequentemente, no aumento do absenteísmo e afastamentos temporários ou permanentes (LIMA et al., 2014).

Conforme Santos et al. (2017), tais condições são fatores preditivos da exposição aos riscos ocupacionais e, como consequência, geram o aumento de afastamentos do trabalho e licenças médicas. Além disso, Moraes et al. (2015) apontam que, como agravo da atividade laboral do enfermeiro, tem-se: (i) atuação por regime de escala, o que inviabiliza uma recuperação gradual do trabalhador; (ii) baixa remuneração, o que influencia a atuação em dupla jornada de trabalho; e (iii) sobrecarga laboral, em caso de absenteísmo de um membro da equipe de trabalho.

\section{Procedimentos Metodológicos}


Estudos científicos caracterizam-se pela utilização de procedimentos metodológicos. O método é um conjunto de atividades sistemáticas que baseiam e permitem atingir os objetivos do trabalho com resultados coerentes (MARCONI e LAKATOS, 2010). A Tabela 1 (um) apresenta o enquadramento da metodologia que foi utilizada para a execução do presente estudo.

Tabela 1 - Enquadramento metodológico

\begin{tabular}{c|l|l}
\hline & Natureza & Básica \\
Classificação da & Método Científico & Indutivo \\
Pesquisa & Abordagem & Qualitativa \\
& Objetivos & Exploratória \\
& Procedimentos Técnicos & Documental \\
\hline
\end{tabular}

Fonte: Adaptado de Marconi e Lakatos (2010) e Gil (2010).

O trabalho quanto a sua finalidade é considerado de natureza básica (GIL, 2010). Em relação ao método científico, a pesquisa é caracterizada como indutiva porque tem como objetivo levar a conclusões, cujo o conteúdo é mais amplo do que as premissas em que se baseou (MARCONI e LAKATOS, 2010). Quanto aos procedimentos técnicos para a elaboração deste estudo, utilizou-se uma pesquisa bibliográfica. Para Marconi e Lakatos (2010), trata-se de pesquisa em que os materiais ainda não receberam um tratamento analítico e que podem ser reelaborados.

Este estudo teve como objetivo verificar quais são os principais problemas biomecânicos relacionados à atividade laboral dos enfermeiros, por meio de uma revisão básica da literatura. Dessa forma, a pesquisa foi gerada por meio das bases de científicas "ScienceDirect", "Scopus", "PubMed" e "Medline". A Figura 1 (um) apresenta como foram relacionadas as palavras-chave, bem como as suas combinações.

Figura 1 - Palavras-chave pesquisadas e combinações

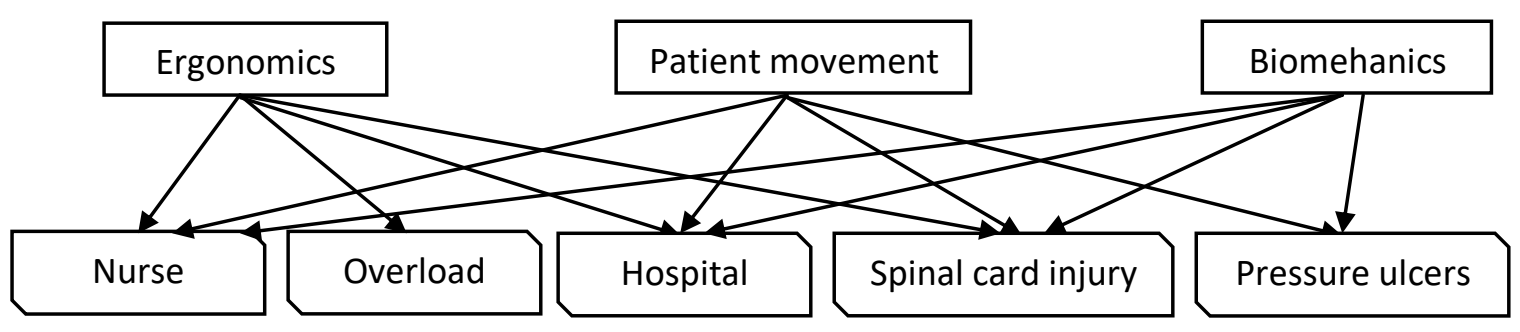

Fonte: autores. 
O período selecionado foi de 2013 a 2018 e, como resultado preliminar, após as relações das palavras-chave, encontrou-se pouco mais de 47 resultados. Desses, através de refinamentos, sete estudos foram selecionados para compor o quadro de artigos referência sobre o tema. A seleção dos artigos, publicados nas bases de dados, foi executada conforme a disponibilidade de acesso da instituição de ensino que os pesquisadores são vinculados. Como fator de restrição da pesquisa, tem-se a seleção de artigos "oppen acess" e com publicações inferiores, no máximo, a cinco anos do ano de 2018.

\section{Resultados e Discussões}

Por meio da revisão da literatura foi possível identificar vários estudos que relatam sobre o uso de dispositivos ergonômicos, que visam auxiliar a redução da carga mecânica dos profissionais da saúde e, principalmente dos enfermeiros nas atividades de manuseio do paciente. No estudo de Drew et al. (2016), por exemplo, é apresentado o uso de lençóis deslizantes que reduzem o atrito do paciente com a cama, como consequência, tem-se a redução do risco de lesão musculoesquelética aos profissionais da saúde, quando comparado aos lençóis de algodão tradicionais, devido à menor compressão da coluna vertebral deles. O Quadro 1 (um) apresenta um conjunto dos estudos mais relevantes sobre os riscos biomecânicos relacionados à atividade laboral dos enfermeiros.

Quadro 1 - Trabalhos mais relevantes: riscos biomecânicos

\begin{tabular}{|c|c|c|c|}
\hline Ano & Título & Periódico & Autoria \\
\hline 2013 & $\begin{array}{l}\text { Establishing a physical therapist-driven } \\
\text { model of safe patient handling and movement } \\
\text { programs in a general hospital }\end{array}$ & Work & Geiger \\
\hline 2013 & $\begin{array}{l}\text { Ergonomics and Nursing in Hospital } \\
\text { Environments }\end{array}$ & Workplace health \& safety & Rogers, et al. \\
\hline 2015 & $\begin{array}{l}\text { Electric Versus Hydraulic Hospital Beds: } \\
\text { Differences in Use During Basic Nursing } \\
\text { Tasks }\end{array}$ & $\begin{array}{c}\text { International Journal of } \\
\text { Occupational Safety and } \\
\text { Ergonomics }\end{array}$ & Capodaglio \\
\hline 2016 & $\begin{array}{l}\text { Biomechanical evaluation and perceived } \\
\text { exertion of a lateral patient-handling task }\end{array}$ & Occupational Ergonomics & Drew et al. \\
\hline 2017 & $\begin{array}{l}\text { Prevalence of musculoskeletal pain in nursing } \\
\text { professionals working in orthopedic setting }\end{array}$ & $\begin{array}{l}\text { Sociedade Brasileira para o } \\
\text { estudo da dor }\end{array}$ & Santos, et al. \\
\hline
\end{tabular}




\begin{tabular}{|c|l|c|c|}
\hline 2017 & $\begin{array}{l}\text { Prevalence and Perceptions of } \\
\text { Musculoskeletal Disorders Among Hospital } \\
\text { Nurses in Pakistan: A Cross-sectional Survey }\end{array}$ & Cureus & $\begin{array}{c}\text { Rathore, Attique e } \\
\text { Asmaa }\end{array}$ \\
\hline 2018 & $\begin{array}{l}\text { An Inspection Tool and Process to Identify } \\
\text { Modifiable Aspects of Acute Care Hospital } \\
\text { Patient Care Units to Prevent Work-Related } \\
\text { Musculoskeletal Disorders }\end{array}$ & Workplace Health \& Safety & Grant et al. \\
\hline
\end{tabular}

Fonte: autores.

Por um viés mais tradicional, o estudo de Geiger (2013) apresenta o uso de equipamentos auxiliares de transferência de pacientes por meio de elevadores e pranchas deslizantes. Tais equipamentos visam prevenir os riscos biomecânicos dos profissionais da saúde, mas a sua utilização é ainda de baixa escala no Brasil. Geiger (2013) também descreve em seu estudo que a utilização de tecnologias que visam auxiliar os trabalhadores em suas tarefas apresentam surpreendentemente um aspecto negativo que foi o aumento à exposição ao estresse psicossocial por parte dos enfermeiros.

O crescimento do nível de estresse do trabalho pode ser consequência da indisponibilidade de treinamento do usuário às novas tecnologias de trabalho, o que gera um aumento da tensão corpórea pela nova atividade laboral. Isso fica comprovado no estudo de Grant et al. (2018), que apresentam uma ferramenta que visa à inspeção e auxilio na identificação dos riscos inerentes aos aspectos modificáveis do ambiente físico do trabalho, tendo em vista a redução de distúrbios osteomusculares relacionados ao trabalho em hospitais.

Rogers et al. (2013) descrevem como as condições do local de trabalho, o ambiente e as atividades laborais podem contribuir para lesões musculoesqueléticas em enfermeiros. Concomitantemente, o estudo de Capodaglio (2015) avaliou, por meio de um estudo de caso, os aspectos biomecânicos, posturais e ergonômicos durante as tarefas reais de assistência ao paciente realizadas por enfermeiros. Tais estudos revelam que esses profissionais estão expostos a exigências excessivas de suas habilidades físicas e cognitivas em vista do ambiente, técnicas e turnos de trabalho.

Nesse contexto, Santos et al. (2017) revelaram por meio de sua pesquisa a prevalência das dores musculoesquelética nos profissionais de enfermagem. Assim, conforme a pesquisa, as regiões anatômicas mais afetadas no caso dessa classe de trabalhadores são: (i) os membros inferiores e superiores das costas; (ii) o pescoço; (iii) os ombros; (iv) os tornozelos/pés e (v) os punhos/mãos. Além disso, por meio desse estudo foi possível identificar que os enfermeiros e os 
técnicos de enfermagem apresentaram maiores prevalências de traumas musculoesqueléticos quando comparado a outras classes de profissionais da área da saúde.

Por conseguinte, Rathore, Attique e Asmaa (2017) complementam descrevendo sobre os principais distúrbios osteomusculares relacionados à atividade laboral dos enfermeiros. Dessa forma, o artigo destaca duas frentes da análise da prevalência dos distúrbios musculoesqueléticos nesses profissionais da saúde: (i) analisou o trabalho direto dos enfermeiros durante um ano, nesse caso, 31,6\% da amostra apresentaram dores na região lombar, ombro e partes superiores das costas; e (ii) analisou as causas de afastamentos do trabalho dos enfermeiros, sendo identificado que 60,6\% da amostra deu-se em função da execução de tarefas que exigiam posturas inadequadas com a necessidade de movimento dos membros superiores.

\section{Considerações Finais}

Por meio deste estudo, foi possível evidenciar as principais causas das incidência de lesões musculoesqueléticas sofridas pelos profissionais da área da saúde, principalmente por parte dos enfermeiros e técnicos de enfermagem. Concomitantemente, o estudo trouxe consigo a relação do alto nível de afastamentos por doenças no trabalho em função do meio de atuação e das características das tarefas executas pelos enfermeiros. $\mathrm{O}$ fator predominante dos afastamentos, nesses profissionais, é a fadiga muscular, ocasionada pela característica multifatorial do trabalho.

Ademais, este estudo apontou a dificuldade dos enfermeiros e técnicos de enfermagem em manter, na execução de suas tarefas laborais, um posicionamento ergonômico adequado por causa na exposição de trabalho vinculada à falta do uso de equipamentos que auxiliam as tarefas que exigem uma carga musculoesquelética incompatível com as capacidades físicas do trabalhador. Aos enfermeiros, conforme pesquisa executada, a principal tarefa causadora de lesões está vinculada ao reposicionamento do corpo de pacientes ao leito.

Além disso, através do estudo, foi possível verificar que atualmente tem-se implementado no ambiente hospitalar equipamentos que auxiliam os profissionais da saúde na movimentação dos pacientes de forma mecânica, o que minimiza os riscos de lesões, tais como: (i) uso de macas e camas com comandos eletrônicos; (ii) elevadores de pacientes; (iii) passantes deslizantes e outros. Mas, para alguns autores, a falta de treinamento e capacitação no uso dos equipamentos que visam auxiliar os serviços dos profissionais da saúde, tais como os elevadores de paciente, pode ser considerado um fator desencadeante de stress funcional, ao invés de mitigar os riscos de 
lesão. Para o sucesso da implementação dessas novas tecnologias, o treinamento dos profissionais deve ser disseminado de forma eficiente.

Conclui-se que no setor da saúde no Brasil, não há ainda uma dispersão em grande escala dos equipamentos que visam prevenir os riscos biomecânicos relacionados à atividade laboral dos profissionais da saúde. Além disso, ressalva-se que o presente estudo trouxe consigo uma contribuição quanto ao esclarecimento dos principais riscos musculoesqueléticos relacionados à execução das tarefas dos profissionais da saúde, no caso enfermeiros. Dessa forma, para trabalhos futuros, sugere-se um estudo de caso em um hospital universitário, para que possa evidenciar o número e as causas dos afastamentos dos enfermeiros e técnicos em enfermagem, no âmbito de um hospital escola no Brasil.

\section{Referências}

AZADEH, A. et al. A unique algorithm for the assessment and improvement of job satisfaction by resilience engineering : Hazardous labs. International Journal of Industrial Ergonomics, v. 49, pp. 68-77, 2015.

AZADEH, A; ROUDI, E.; SALEHI, V. Optimum design approach based on integrated macro-ergonomics and resilience engineering in a tile and ceramic factory. Safety Science, v. 96, pp. 62-74, 2017.

BAE, S.H., FABRY, D. Assessing the relationships between nurse work hours/ overtime and nurse and patient outcomes: systematic literature review. Nurs. Outlook, v. 62 (2), pp. 138e156, 2014.

BRASIL. Ministério da Previdência Social. Instrução normativa INSS/DC n ${ }^{\circ}$ 98, de 5 de dezembro de 2003. Aprova Norma Técnica sobre Lesões por Esforços Repetitivos-LER ou Distúrbios Osteomusculares Relacionados ao Trabalhador. Brasília: Ministério da Previdência Social; 2003.

BRASIL. Ministério do Trabalho e Emprego. Disponível em: http://www.brasil.gov.br/economia-eemprego/2017/04/dor-nas-costas-e-a-maior-causa-de-afastamento-do-trabalho Acesso em: 19 de jul. 2018.

CHANCHAI, W. et al. The impact of an ergonomics intervention on psychosocial factors and musculoskeletal symptoms among Thai hospital orderlies. International Journal of Environmental Research and Public Health, v. 13, n. 5, pp. 1-10, 2016.

CHOI, S. D., BRINGS, K. Work-related musculoskeletal risks associated with nurses and nursing assistants handling overweight and obese patients: a literature review. Work, v. 53, pp. 439-448, 2016.

D'ERRICO, A., et al. Low back pain and associated presenteeism among hospital nursing staff. Journal Occup Health, v. 55,p p. 276-283, 2013.

D'ERRICO, A., et al. Risk factors for upper extremity musculoskeletal symptoms among call center employees. Journal Occup. Health, v. 52, pp.115-124, 2010.

DANIELL, N.; MERRETT, S.; PAUL, G. Effectiveness of powered hospital bed movers for reducing physiological strain and back muscle activation. Applied Ergonomics, v. 45, n. 4, pp. 849-856, 2014.

DOUGHERTY, L., LISTER, S. Moving and positioning. In: Dougherty. The royal marsden hospital manual of clinical nursing procedures. Ed. Wiley-Blackwell, West Sussex, UK, pp. 279-332, 2011.

DREW, K. E., KOZEY, J. W., MORSIDE, J. M. Biomechanical evaluation and perceived exertion of a lateral patient-handling task. Occup. Ergon. v. 12 (4), pp. 151-163, 2016. 
DUL, J., NEUMANN, W. Ergonomics contributions to company strategies. Appl Ergon, v. 40 (4), pp. 745-752, 2009.

GEIGER, J. S. Establishing a physical therapist-driven model of safe patient handling and movement programs in a general hospital. Work, v. 45, n. 2, pp. 147-160, 2013.

GRANT, M. P. et al. An inspection tool and process to Identify modifiable aspects of acute care hospital patient care units to prevent work-related musculoskeletal disorders. Workplace Health \& Safety, v. XX, n. In press, p. 1-15, 2017.

HARCOMBE, H., et al. Physical and psychosocial risk factors for musculoskeletal disorders in New Zealand nurses, postal workers and office workers. Inj Prev, v. 16, pp. 96-100, 2010.

HEGEWALD, J. et al. Do technical aids for patient handling prevent musculoskeletal complaints in health care workers? - A systematic review of intervention studies. International Journal of Environmental Research and Public Health, v. 15, n. 3, 2018.

HENDRICK, H.W. Applying ergonomics to systems: Some documented "lessons learned". Applied Ergonomics, v. 39, n. 4, pp. 418-426, 2008.

HENDRICK, H.W. Ergonomics in organizational design and management. Ergomics, 1991, v 34, n. 6, pp. 743-756.

HENDRICK, H.W.; KLEINER, B.M. Macro-Ergonomics: Theory, Methods, and Applications. Lawrence Erlbaum Associates, Publishers, Mahwah, 2002.

HIGNETT, S. et al. Human factors and ergonomics and quality improvement science: Integrating approaches for safety in healthcare. BMJ Quality and Safety, v. 24, n. 4, pp. 250-254, 2015.

HOLLNAGEL, E. FRAM: the functional resonance analysis method modeling complex socio-technical systems. Burlington: Ashgate; 2012.

IEA. The discipline of ergonomics. Disponível em: 〈www.iea.cc〉. Acesso em: 20, set. 2017.

IIDA, I.; BUARQUE, L. Ergonomia: projeto e produção. 3. ed. São Paulo: Edgard Blucher, 2016. 850p.

KAJIKI, S. et al. A randomized controlled trial of the effect of participatory ergonomic low back pain training on workplace improvement. Journal of Occupational Health, v. 59, n. 3, p. 256-266, 2017.

KARSH, B.; WATERSON, P.; HOLDEN, R.J. Crossing levels in systems ergonomics: A framework to support mesoergonomic'inquiry. Applied ergonomics, 2013.

LEE, K. A., DZIADKOWIEC, O., MEEK, P. A systems science approach to fatigue management in research and health care. Nursing Outlook, v. 62 (5), p. 313-321, 2014.

LIMA, A. C., et al. Fatores associados à dor musculoesquelética em trabalhadores de enfermagem hospitalar. Revista de Enfermagem da UERJ, v. 22(4), p. 526-32, 2014.

MAO, X. et al. An evaluation of the effects of human factors and ergonomics on health care and patient safety practices: A systematic review. Plos One, v. 10, n. 6, pp. 1-19, 2015.

MARCONI, M. A.; LAKATOS, E. M. Fundamentos da metodologia científica. São Paulo: Altas, 320 p., 2010.

MBADA, C. E., et al.: Work-related musculoskeletal disorders among health workers in a Nigerian teaching hospital. TAF Prev Med Bull, p.11:583-8, 2012.

MOAZZAMI, Z. et al. Effect of an Ergonomics-Based Educational Intervention Based on Transtheoretical Model in Adopting Correct Body Posture Among Operating Room Nurses. Global Journal of Health Science, v. 8, n. 7, p. $26,2015$. 
MORAES, K. N., et al. Fatores relacionados ao absenteísmo por doença em profissionais de enfermagem: uma revisão integrativa. Gestão e Saúde, v. 6 (1), p. 565-90, 2015.

OKUNRIBIDO, O. O.; MAGNUSSON, M.; POPE, M. H. The role of whole body vibration, posture and manual material handling as risk factors for low back pain in occupational drivers. Ergonomics, v. 51, pp. 308-329, 2008.

PARENT-THIRION, A., et al. Fifth European Working Conditions Survey. Overview report. Dublin(Ireland): Eurofound, Report,160 pp., 2012.

PAULA, E. A. et al. Qualidade de vida de trabalhadores com LER/DORT e lombalgia ocupacional atendidos no Cerest de Guarulhos, São Paulo. Revista Brasileira de Saúde Ocupacional, v. 41, n. 0, p. 1-11, 2016.

PUNNETT, L. et al. Estimating the global burden of low back pain attributable to combined occupational exposure. Am J Ind Med, v. 48, pp. 459-469, 2005.

RAMÓN, A. et al. DE ENFERMERÍA: REVISIÓN SISTEMÁTICA Y META-ANÁLISIS Occupational Exposure to Psychosocial Factors and Presence of Musculoskeletal disorders in Nursing Staff: A review of Studies and MetaAnalysis trastornos musculoesqueléticos ( TME ) son alteraciones. Rev. Esp. Salud Pública, v. 91, p. 1-27, 2017.

RATHORE, F. A.; ATTIQUE, R.; ASMAA, Y. Prevalence and Perceptions of Musculoskeletal Disorders Among Hospital Nurses in Pakistan: A Cross-sectional Survey. Cureus, v. 9, n. 1, pp. 1-10, 2017.

ROGERS, B.; BUCKHEIT, K.; OSTENDORF, J. Ergonomics and Nursing in Hospital Environments. Workplace Health \& Safety, v. 61, n. 10, pp. 429-439, 2013.

ROQUELAURE, Y. Promoting a Shared Representation of Workers' Activities to Improve Integrated Prevention of Work-Related Musculoskeletal Disorders. Safety and Health at Work, v. 7, n. 2, pp. 171-174, 2016.

SANTOS, E. C. DOS et al. Prevalence of musculoskeletal pain in nursing professionals working in orthopedic setting. Revista Dor, v. 18, n. 4, p. 298-306, 2017.

STEEGE, L. M., et al. Evaluation of physically and mentally fatiguing tasks and sources of fatigue as reported by registered nurses. J. Nurs. Manag, v. 23 (2), pp. 179-189, 2015.

STEEGE, L. M.; DYKSTRA, J. G. A macroergonomic perspective on fatigue and coping in the hospital nurse work system. Applied Ergonomics, v. 54, pp. 19-26, 2016.

SZETO, G. P. Y., et al. Surgeons' static posture and movement repetitions in open and laparoscopic surgery. Journal Surg. Res, pp. 172:19-31, 2012.

TORTORELlA, G. L.; VERGARA, L. G. L.; FERREIRA, E. P. Lean manufacturing implementation: an assessment method with regards to socio-technical and ergonomics practices adoption. International Journal of Advanced Manufacturing Technology, v. 89, pp. 3407-3418, 2017.

VANDERGRIFT, J. L. et al. Physical and psychosocial ergonomic risk factors for low back pain in automobile manufacturing workers. Occupational and Environmental Medicine, v. 69, n. 1, pp. 29-34, 2012.

VANDERGRIFT, J. L., et al. Physical and psychosocial ergonomic risk factors for low back pain in automobile manufacturing workers. Occup. Environ. Med, v. 69, pp. 29-34, 2012.

WATERS, T. R., et al. A cross-sectional study of risk factors for musculoskeletal symptoms in the workplace using data from the General Social Survey (GSS). J Occup Environ Med, v. 49, pp.172-184, 2007.

WATERS, T., NELSON, A., PROCTOR, C. Patient handling tasks with high risk for musculoskeletal disorders in critical care. Crit. Care Nurs. Clin. North Am, v. 19, pp. 131-143, 2007.

WILLIAMSON, A., FRISWELL, R. Fatigue in the workplace: causes and countermeasures. Fatigue: Biomedicine. Health \& Behav, v. 1, pp. 81-98, 2013. 
WOODS, D. Four concepts for resilience and their implications for systems safety in the face of complexity. in press. Special Issue on Resilience Engineering. Reliab Eng Syst Saf, 2015. 